OPEN ACCESS

Edited by:

José Mordoh,

Fundación Instituto Leloir,

Argentina

Reviewed by:

Luuk Hilbrands,

Radboud University Nijmegen,

Netherlands

Elena Monica Borroni,

Humanitas Research

Hospital, Italy

*Correspondence:

Marina I. Garin

marina.garin@ciemat.es

tThese authors have contributed

equally to this work.

₹These authors share senior authorship.

Specialty section:

This article was submitted

to Vaccines and

Molecular Therapeutics,

a section of the journal

Frontiers in Immunology

Received: 16 September 2016 Accepted: 15 May 2017

Published: 08 June 2017

Citation:

Lopez-Santalla M, Mancheño-

Corvo P, Escolano A, Menta R, DelaRosa O, Abad JL, Büscher D,

Redondo JM, Bueren JA,

Dalemans W, Lombardo E and Garin MI (2017) Biodistribution and Efficacy of Human Adipose-Derived Mesenchymal Stem Cells Following

Intranodal Administration in Experimental Colitis.

Front. Immunol. 8:638. doi: 10.3389/fimmu.2017.00638

\section{Biodistribution and Efficacy of Human Adipose-Derived Mesenchymal Stem Cells Following Intranodal Administration in Experimental Colitis}

\author{
Mercedes Lopez-Santalla ${ }^{1,2+}$, Pablo Mancheño-Corvo ${ }^{3 \dagger}$, Amelia Escolano 4 , \\ Ramon Menta ${ }^{3}$, Olga DelaRosa ${ }^{3}$, Jose Luis Abad ${ }^{5}$, Dirk Büscher ${ }^{6}$, Juan M. Redondo ${ }^{4}$, \\ Juan A. Bueren ${ }^{1,2}$, Wilfried Dalemans ${ }^{7}$, Eleuterio Lombardo ${ }^{3 \neq}$ and Marina I. Garin ${ }^{1,2 * \neq}$ \\ ${ }^{1}$ Division of Hematopoietic Innovative Therapies, Centro de Investigaciones Energéticas, Medioambientales y Tecnológicas \\ (CIEMAT), Centro de Investigación Biomédica en Red de Enfermedades Raras (CIBER-ER), Madrid, Spain, ${ }^{2}$ Advanced \\ Therapies Mixed Unit, Instituto de Investigación Sanitaria Fundación Jiménez Díaz (IIS-FJD), Madrid, Spain, ${ }^{3}$ TiGenix SAU, \\ Madrid, Spain, ${ }^{4}$ Gene Regulation in Cardiovascular Remodeling and Inflammation Laboratory, Centro Nacional de \\ Investigaciones Cardiovasculares Carlos III (CNIC), Madrid, Spain, ${ }^{5}$ Coretherapix, Madrid, Spain, ${ }^{6}$ Grifols, Barcelona, Spain, \\ ${ }^{7}$ TiGenix NV, Leuven, Belgium
}

Mesenchymal stem cells (MSCs) have a large potential in cell therapy for treatment of inflammatory and autoimmune diseases, thanks to their immunomodulatory properties. The encouraging results in animal models have initiated the translation of MSC therapy to clinical trials. In cell therapy protocols with MSCs, administered intravenously, several studies have shown that a small proportion of infused MSCs can traffic to the draining lymph nodes (LNs). This is accompanied with an increase of different types of regulatory immune cells in the LNs, suggesting the importance of migration of MSCs to the LNs in order to contribute to immunomodulatory response. Intranodal (IN), also referred as intralymphatic, injection of cells, like dendritic cells, is being proposed in the clinic for the treatment of cancer and allergy, showing that this route of administration is clinically safe and efficient. In this study, we investigated, for the first time, the biodistribution and the efficacy of Luciferase ${ }^{+}$adipose-derived MSCs (Luci-eASCs), infused through the inguinal LNs (iLNs), in normal mice and in inflamed mice with colitis. Most of the LucieASCs remain in the iLNs and in the adipose tissue surrounding the inguinal LNs. A small proportion of Luci-eASCs can migrate to other locations within the lymphatic system and to other tissues and organs, having a preferential migration toward the intestine in colitic mice. Our results show that the infused Luci-eASCs protected $58 \%$ of the mice against induced colitis. Importantly, a correlation between the response to eASC treatment and a higher accumulation of eASCs in popliteal, parathymic, parathyroid, and mesenteric LNs were found. Altogether, these results suggest that IN administration of eASCs is feasible and may represent an effective strategy for cell therapy protocols with human adipose-derived MSCs in the clinic for the treatment of immune-mediated disorders.

Keywords: adipose-derived mesenchymal stem cells, intranodal therapy, colitis, biodistribution, efficacy, immunomodulation 


\section{INTRODUCTION}

Mesenchymal stem cells (MSCs) are multipotent adult stem cells capable of self-renewal and differentiation into multiple cell lineages of mesodermal origin such as chondrocytes, adipocytes, and osteocytes (1). These cells have been identified in multiple tissues such as bone marrow (2), umbilical cord blood (3), amniotic fluid (4), adipose tissue (5), dental pulp (6), and among others. MSCs are considered as a novel modality of therapy for a wide variety of degenerating and immunological disorders $(1,2,7,8)$. The encouraging results in animal models have initiated the translation of MSC therapy in clinical trials in a range of immunomediated diseases such as graft versus host disease (9), inflammatory bowel disease (10-12), multiple sclerosis (13), systemic lupus erythematosus $(14,15)$, rheumatoid arthritis (16-20), and among others. The immunomodulatory capacity of MSCs takes place both by direct cell-to-cell contact and by means of soluble factors (21). At present, it is unknown whether the therapeutic effects of MSCs are carried out by a small fraction of the administered MSCs that migrates to sites of inflammation or/and through distant induction of regulatory immune cells that mediate immunomodulation and tissue repair (22). There are controversial data regarding the presence of MSCs at site of inflammation (23-25). In any case, their therapeutic effects are linked to a final increase of myeloid and lymphoid cells with a regulatory phenotype in the secondary lymphoid tissues associated to the inflammation site $(18,19,25,26)$. Thus, in this study (27), we hypothesized that intranodal (IN) administration of MSCs could be feasible and would represent an alternative route for modulating immune responses $(18,25,28)$. IN administration treatments are being extensively used in clinical trials for the treatment of cancer (29-32) and allergy $(33,34)$, indicating that this route of administration is feasible in humans and can be applied to the clinic. Data from clinical trials show that IN therapy is easy, practically painless, safe and is associated with a low risk of systemic adverse effects (35-37). Additionally, Gil-Ortega et al. provided robust evidences that endogenous adipose-derived MSCs (eASCs) can navigate through the lymphatic system (28). At present, IN administration of MSCs has not been assessed previously, whereas intravenous administration of MSCs has been used in many experimental models and in clinical trials. However, the intravenous injection of MSCs is thought to be limited by cell entrapment in the lungs $(24,38)$. This might limit the bioavailability of the cells, their trafficking to tissues and, possibly, their therapeutic effects (24). Local administration of MSCs is of interest in situations where they can be directly implanted in the injured tissues trying to improve their beneficial effects (39-42). The number of MSCs that can be delivered inside the lymph node (LN) after IN administration could be significantly greater than the number of cells that reach the LNs following their delivery through the blood stream, either through intravenous or intraperitoneal administration. This may allow achieving robust therapeutic effects while potentially reducing the cell dose of MSCs required for modulating inflammation.

In this study, we aimed to examine the biodistribution of human Luciferase ${ }^{+}$eASCs (Luci-eASCs) after IN administration in steady state and following an acute inflammatory challenge in the colon with the haptenizing reagent trinitrobenzene sulfonic acid (TNBS). Strikingly, modulation of intestinal inflammation was observed. In parallel to this study, we also demonstrate that IN infusion of eASCs can modulate ongoing acute inflammatory responses in an experimental model of collagen-induced arthritis (27).

\section{MATERIALS AND METHODS}

\section{Mice}

C57/BL6 male mice of 6-8 weeks of age were obtained from Charles River (Burlington, MA, USA). All experiments were performed in accordance with the corresponding regulations regarding experimental animal welfare (RD 223/1998 and Directive 2010/63/EU protocols).

\section{Generation of Human Expanded Adipose- Derived MSCs}

Human samples were obtained after informed consent as approved by the Spanish Ethics Committee of reference for the site of tissue procurement (Clínica de la Luz Hospital, Madrid, Spain). Human adipose tissue aspirates from healthy donors were processed as described elsewhere (18). All the eASCs used fulfilled the release criteria of identity, purity, and potency needed for their clinical use.

\section{Generation of Luci-eASCs}

Reporter Luciferase-EGFP bicistronic retroviral vector was constructed using standard cloning procedures. The H2B gene was amplified by PCR (forward, TATGGGTACCGCCACCATGCCA GAGCCAGCGAAG; reverse, TATGGATCCTTAGCGCTGGTG TACTTG) and cloned into pCopGFP2i-N (Evrogen, Moscow, Russia) using Acc65I-BamHI sites. Internal ribosome entry site (IRES) element was isolated from pIRES2-EGFP (Clontech, Mountain View, CA, USA) using SalI-MscI and cloned upstream H2B-CopGFP2i into SalI-Acc65I (Klenow blunted) sites. Luciferase was cut from pGL2 Basic (Promega, Madison, WI, USA), using XhoI-XbaI (Klenow blunted) restriction sites, and inserted upstream IRES-H2B-CopGFP2i into XhoI-SalI (Klenow blunted). CopGFP2i was then replaced by EGFP, from pEGFP-N1 (Clontech) using AgeI-NotI sites. The LuciferaseIRES-H2B-EGFP cassette was separated using BglII-NotI and cloned into BamHI-NotI sites of pRV retroviral vector to give the final pRV-Luciferase-IRES-H2B-EGFP bicistronic reporter construction. Transfection and generation of viral supernatants were performed using polyethylenimine (1 vol PEI:2 vol DNA), Viral supernatants were used for infection of eASCs. For infection, eASCs were seeded in 6-well plates and infected with retroviral particles concentrated when cell confluency was between 70 and $80 \%$. Finally, the transduction was evaluated by flow cytometry (FACS Calibur ${ }^{\mathrm{TM}}, \mathrm{BD}$ Bioscience, San Diego, CA, USA), selecting clones with $90 \%$ expression of EGFP by cell sorting.

\section{Immunophenotyping of Luci-eASCs}

Luci-eASCs were defined according to the criteria of the International Society for Cellular Therapy (43). Luci-eASCs were 
characterized by their immunophenotype; positive for CD73 (AD2), CD90 (5E10, both from Becton Dickinson), and CD105 (43A3, from Biolegend) and negative for CD14 (RM052, from Immunotech), CD34 (8G12, from Becton Dickinson), CD45 (J33, from Beckman Coulter), and HLA-DR (AF6-120.1, from eBiosciences).

\section{Immunosuppression Assay of Luci-eASCs}

Buffy coats were provided by the National Transfusion Centre of the Comunidad Autonoma of Madrid. Peripheral blood mononuclear cells (PBMCs) were isolated by density centrifugation gradient using Ficoll-Paque Plus (GE Healthcare Biosciences AB, Uppsala, Sweden) following the supplier's protocol. Purity was verified by flow cytometry.

For CFSE labeling, PBMCs were washed extensively to remove fetal bovine serum (FBS), resuspended in a $10 \mu \mathrm{M}$ CFSE (Carboxyfluorescein diacetate $\mathrm{N}$-succinimidyl ester, SigmaAldrich, St. Louis, MO, USA) solution ( $10^{7}$ PBMC per $200 \mu \mathrm{L}$ of solution), and incubated under constant shaking at $37^{\circ} \mathrm{C}$ for $10 \mathrm{~min}$. Reaction was stopped by adding ice-cold medium $(\mathrm{RPMI}+10 \% \mathrm{FBS})$, and cells were washed three times with icecold phosphate buffer saline. Cells were then cultured overnight and one aliquot was used to set up and control the FL-1 voltage for CFSE. After resting overnight, CFSE-labeled PBMCs were activated with the Pan T Cell Activation Kit (microbeads coated with anti-CD3, anti-CD2, and anti-CD28; Miltenyi Biotech, Auburn, CA, USA) following the manufacturer's instructions. PBMCs $\left(10^{6}\right.$ cells/well $)$ were cultured in 24 -well plates alone or with Luci-eASCs $\left(4 \times 10^{4}\right.$ cells/well; Luci-eASC:PBMC ratio $1: 25)$ in a total volume of $1 \mathrm{~mL}$ of RPMI $+10 \%$ FBS. The 1:25 ratio was chosen because it provided a high inhibitory effect, on the basis of previous studies $(44,45)$. After 5 days, PBMCs were harvested and labeled with 7-AAD and anti-CD3 antibody, and cell proliferation of the $\mathrm{CD}^{+} / 7-\mathrm{AAD}^{-}$population (viable $\mathrm{CD} 3$ T lymphocytes) was determined by flow cytometry, according to loss of CFSE signal. Data were analyzed with the use of FCSExpress 4 (De Novo Software, Glendale, CA, USA) and BD CellQuest ${ }^{\mathrm{TM}}$ Pro analysis (Becton Dickinson) softwares. CaliBRITE beads (BD Bioscience, Erembodegem-Aalst, Belgium) were used before each assay to calibrate the cytometer.

\section{Induction and Evaluation of Colitis after Treatment with Luci-eASCs}

To induce colitis, male C57BL/6 mice (6-8 weeks old) were preimmunized on their shaved back with $1 \%$ of TNBS (Sigma-Aldrich, St. Louis, MO, USA) in a mixture of acetone:olive (4:1 v/v). After 1 week, colitis was induced by intrarectal administration of $3 \mathrm{mg}$ of TNBS in $50 \%$ ethanol $(100 \mu \mathrm{L})$ per mouse. $1 \mathrm{~h}$ after TNBS administration, $3.2 \times 10^{5}$ of Luci-eASCs per mouse were administered IN, by injection of the cell suspension in the iLNs $\left(1.6 \times 10^{5}\right.$ cells in both the right and the left iLNs). As controls, healthy mice infused with the Luci-eASC and mice treated with intrarectal 50\% EtOH only (vehicle of the TNBS) and infused with Luci-eASC IN were used. Score of colitis (weight of mice, stools, and general aspect of mice) was monitored for $48 \mathrm{~h}$.

\section{In Vivo Optical Bioluminescence Imaging}

Bioluminescent imaging analysis was conducted at $48 \mathrm{~h}$ after the infusion of Luci-eASCs with the IVIS 200 imaging system (Caliper, Hopkinton, MA, USA).

Whole-body bioimaging analysis was done in anesthetized mice. The bioimaging analysis in main tissues and organs (liver, spleen, intestine, lungs, heart, and blood), secondary LNs (popliteal, popLN; parathymic, thymLN; parathyroid, thyrLN; mesenteric, mLN; caudal, cLN; axillary, axLN; and inguinal, iLN, LNs) and the adipose tissue around the inguinal LNs, where the Luci-eASCs were injected, were determined immediately after culling the mice. Photons emitted were acquired as photons per $\mathrm{s} / \mathrm{cm}^{2}$ per steradian using Living Imaging 3.0 (Caliper). For photon quantification, a region of interest was manually selected and kept constant within each experiment.

Bioluminescence signal was analyzed as percentage of light units per tissue relative to the total number of light units per mouse. Total bioluminescence signal for LNs, tissues, and organs were calculated as the sum of the light units in each tissue. Values of bioluminescence signal below 10,000 light units were considered negative.

\section{Statistical Analysis}

Data are presented as the interquartile range (p75, upper edge; p25, lower edge; p50, midline; p95, line above the box, and p5, line below the box). Normal distribution was analyzed by the Shapiro-Wilks test. Non-parametric techniques (Mann-Whitney $U$ test) were used (with Bonferroni adjustment). Analysis was performed using the software Stata 11 (StataCorp, USA) and GraphPad Prism 5.00 for Windows program (GraphPad Software, San Diego, CA, USA).

\section{RESULTS}

\section{The Majority of the Intranodally Injected Luci-eASCs Remain in the LNs $48 \mathrm{~h}$ Postinfusion}

In the present study, we investigated the biodistribution of the Luci-eASCs after IN administration in an experimental model of acute intestinal inflammation induced by TNBS which displays human inflammatory bowel disease-like clinical, histopathologic, and immunologic features (46).

Human adipose-derived MSCs expressing the luciferase gene were generated by using retrovirus-based methods (See Materials and Methods). To confirm the ASC characteristics of the transduced Luci-eASCs, immunophenotyping by flow cytometry and in vitro immunosuppression assays were carried out. As expected, Luci-eASCs were positive for CD73, CD90, and CD105 and negative for CD14, CD34, CD45, and HLA-DR according to the criteria of the International Society for Cellular Therapy (43) (Figure S1A in Supplementary Material). To evaluate the immunosuppression capacity of Luci-eASCs, T-cell proliferation assays were carried out with CFSE-labeled PBMCs stimulated with microbeads coated with anti-CD3/CD2/CD28 antibodies in the presence or absence of untransduced eASCs or Luci-eASCs (ratio eASCs:PBMCs, 
1:25). Proliferation of viable $\mathrm{CD}^{+} \mathrm{T}$ cells was determined at $120 \mathrm{~h}$ by flow cytometry. Luci-eASCs inhibited T-cell proliferation at similar levels as the untransduced eASCs (Figure S1B in Supplementary Material).

Initial experiments were set up to define for how long the bioluminescence signal of the infused Luci-eASCs can be detected in vivo. A total of $3.2 \times 10^{5}$ Luci-eASCs were injected through the inguinal LNs into healthy mice. The bioluminescence signals were monitored at different time points. As shown in Figure S2A in Supplementary Material, the bioluminescence signal declined very rapidly within the first $72 \mathrm{~h}$ period from the infusion of the Luci-eASCs. Therefore, $48 \mathrm{~h}$ postinfusion of Luci-eASCs was chosen as the best time-point that allows analyzing in vivo the biodistribution of the infused injected Luci-eASCs.

At $48 \mathrm{~h}$ postinfusion of the Luci-eASCs, the highest bioluminescence signals were measured in the LNs analyzed in this study (popLN, thymLN, thyrLN, mLN, cLN, axLN, and iLN), both in healthy [92.0 (70.3-99.2)\%] and in TNBS-colitic mice [57.5 (46.8-79.6)\%], whereas lower levels of the bioluminescence signals were detected in other organs and tissues analyzed (liver, spleen, intestine, lungs, heart, and blood) in both healthy [0.6 (0.3-2.6)\%] and TNBS-colitic mice [2.8 $(0.8-5) \%$ ] (Figure 1; Figure S2 in Supplementary Material). Interestingly, in TNBS-colitic mice, a significant increase of the bioluminescence signal in the tissues and organs studied was detected in comparison with healthy mice. This paralleled with a decrease in the bioluminescence signal in the LNs of TNBS-colitic mice when compared to healthy mice (Figure 1). These results show that the majority of the infused Luci-eASCs remain in the LNs, $48 \mathrm{~h}$ postinfusion and that the inflammatory challenge with TNBS, enhance the trafficking of a small, but significant, proportion of the infused-Luci-eASCs to other tissues and organs.

\section{Intranodal Administered Luci-eASCs Remained in the iLNs and, Upon Inflammation, a Small Proportion of Luci- eASCs Traffics to the Intestine}

To further dissect the biodistribution of the Luci-ASCs and how an acute inflammatory challenge may impact their homing toward the inflamed tissues, the bioluminescence signals were analyzed separately in the LNs (inguinal, popliteal, thymic, thyroid, mesenteric, caudal, and axillary LNs) and in the tissues and organs (liver, spleen, intestine, lung, heart, and peripheral blood). As shown in Figure 2A and Figure S2C in Supplementary Material, when Luci-eASCs were administered IN, most of the bioluminescence signal was found in the iLNs [87.5 (61.5-93.2)\% in healthy mice and $51.4(42.3-68.1) \%$ in TNBS-colitic mice, $p=0.0011]$ where the Luci-eASCs were injected. In contrast, very low levels of the bioluminescence signals [0.4 (0.2-1.3)\%] were measured within the lymphatic system with no clear preference for any of the LNs analyzed (Figure 2A).

Moreover, high bioluminescence signals were also detected in the adipose tissue surrounding the iLNs [5.9 (0.3-35.9)\% in healthy mice and $37.4(11.9-46.7) \%$ in TNBS-colitic mice,

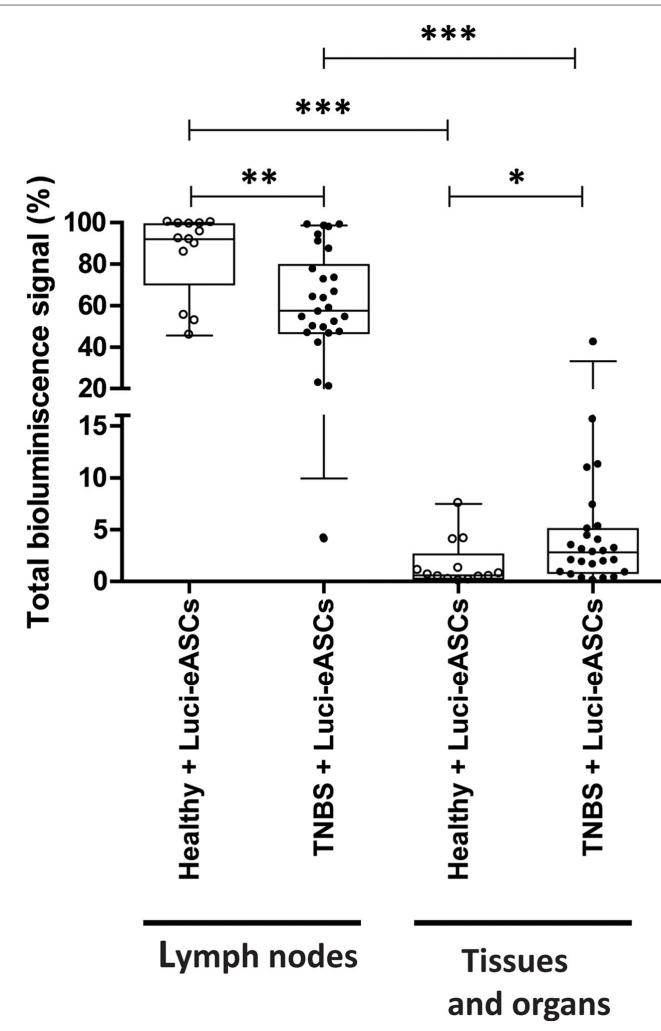

FIGURE 1 | Analysis of total bioluminescence signal of Luci-eASCs in lymph nodes ( $L N s)$ and in tissues and organs at $48 \mathrm{~h}$ following intranodal administration. Sums of bioluminescence signals measured at $48 \mathrm{~h}$ as percentage of light units per LN (inguinal, popliteal, parathymic, parathyroid, mesenteric, caudal, and axillary included) and per tissue and organ (liver, spleen, intestine, lungs, heart, and blood included) relative to the total number of light units per mouse were expressed. Data are presented by dots and box-plots that represent the interquartile range (p75, upper edge; $p 25$, lower edge; p50, midline; p95, line above the box; and p5, line below the box) of the percentage of total bioluminescence signal. Significance was analyzed by the Mann-Whitney $U$ test and represented by ${ }^{*} p<0.05$, ${ }^{* *} p<0.01$, and ${ }^{\star * *} p<0.001$. Results correspond to four independent experiments.

Figure 2B; Figure S2D in Supplementary Material]. This suggests that after IN administration of the Luci-eASCs, the majority of the cells remained at the injection site either inside the LNs or in the adipose tissue associated to the LNs.

The bioluminescence signals in the other tissues and organs analyzed were very low (below 3\%). The bioluminescence signal found in the intestine was significantly increased in TNBScolitic mice $[1.5(0.5-4) \%]$ in comparison with the healthy mice [0.3 (0.1-0.7)\%, $p=0.0037$ ]. Increased bioluminescence signals, but not significant, were found in the liver [0.28 (0.04-0.80)\%], spleen $[0.10(0.01-0.27) \%]$, lungs $[0.10(0.02-0.30) \%]$, and blood [0.14 (0.03-0.30)\%] of the TNBS-colitic mice in comparison with the healthy mice $[0.050(0.008-0.531) \%, 0.05$ (0.02-0.35)\%, $0.04(0.01-0.12) \%$, and 0.009 (0.000-0.065), respectively, Figure 2C; Figure S2D in Supplementary Material]. Additionally, the bioluminescence signal found in the intestine [1.5 (0.5-4)\%] was significantly increased in comparison with the other tissues and organs analyzed in the TNBS-colitic mice 
[0.28 (0.04-0.80)\% for liver, $0.10(0.01-0.27) \%$ for spleen, 0.10 (0.02-0.30)\% for lungs, $0.038(0.001-0.305) \%$ for heart, and 0.14 (0.03-0.30)\% for blood].
Overall, these data indicate that when the Luci-eASCs were administered IN, the majority of the cells remained at the site of injection, either in the iLNs or the adipose tissue adjacent the

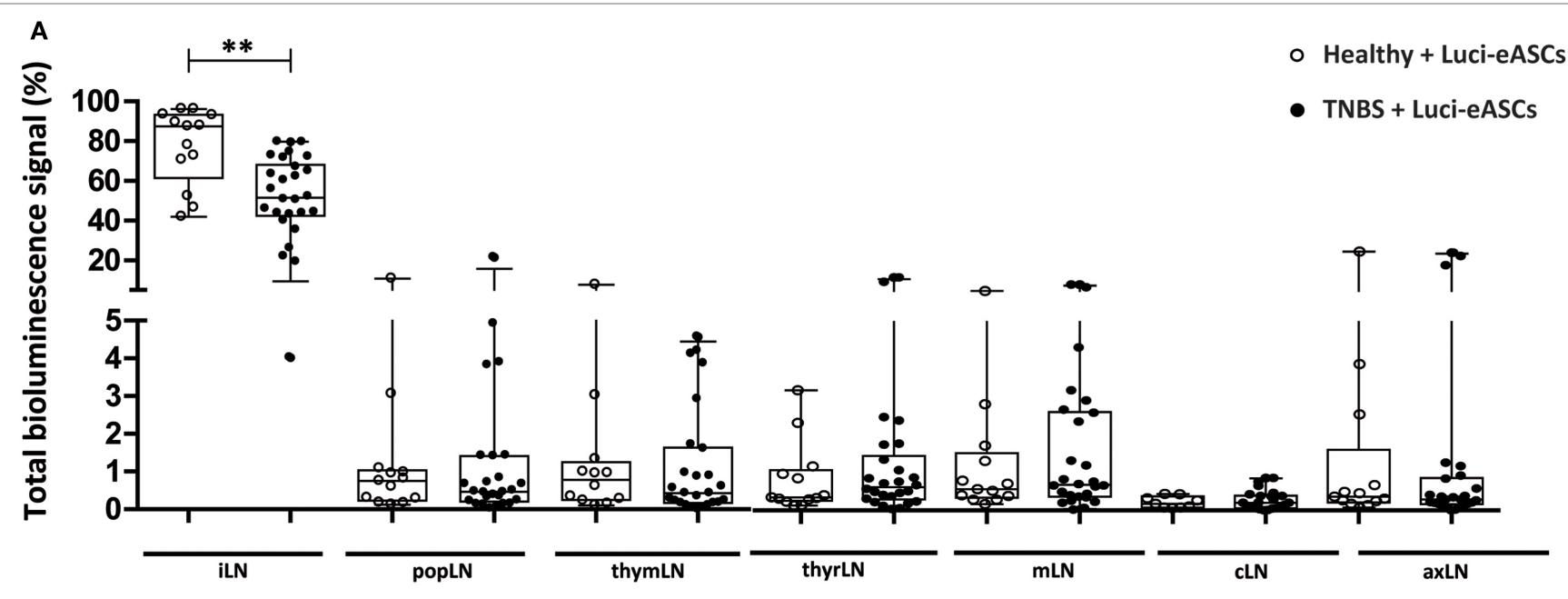

B

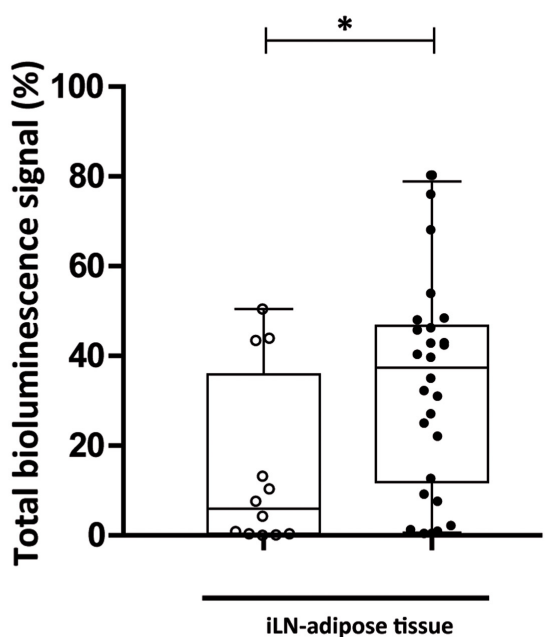

C

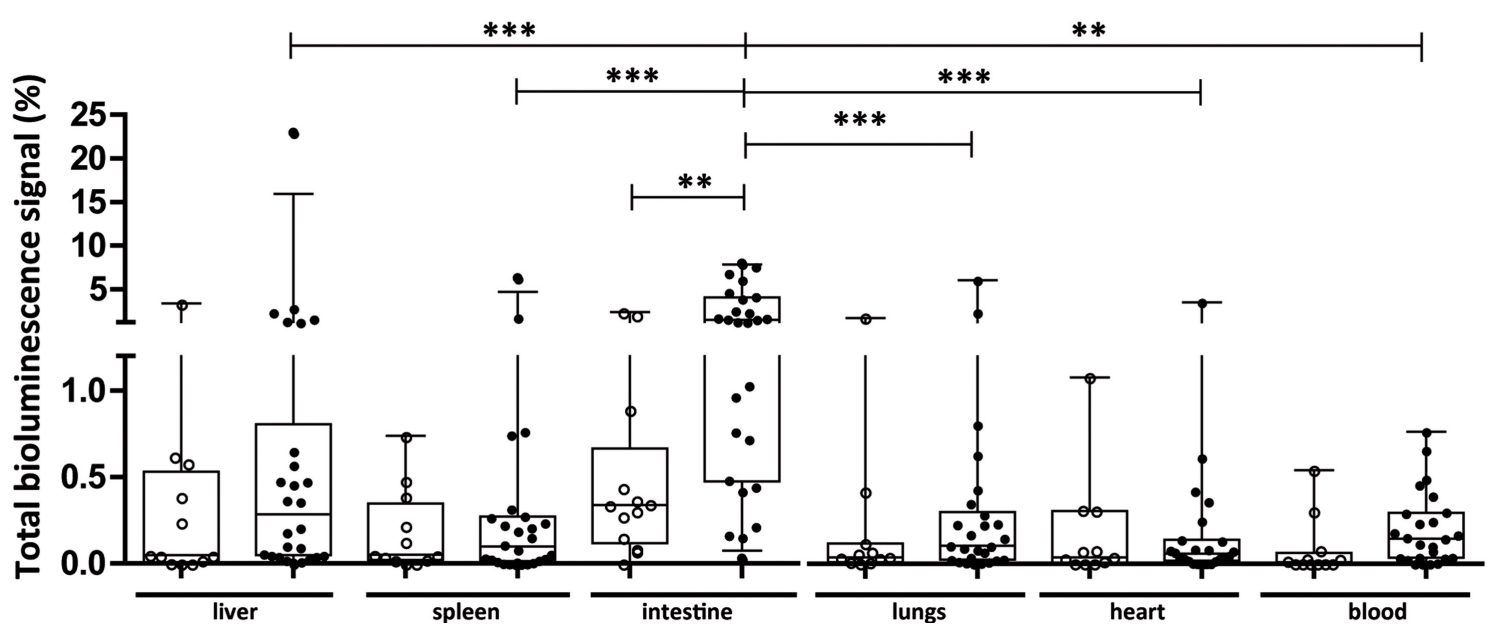


FIGURE 2 | Continued

Analysis of total bioluminescence signal of Luci-eASCs in the different lymph nodes (LNs) (inguinal, popliteal, parathymic, parathyroid, mesenteric, caudal, and axillary), adipose tissue adjacent the inguinal LNs and main tissues and organs (liver, spleen, intestine, lungs, heart, and blood) at $48 \mathrm{~h}$. Bioluminescence signals measured at $48 \mathrm{~h}$ in the inguinal $L N s$ (iLN), popliteal (popLN), parathymic (thymLN), parathyroid (thyrLN), mesenteric (mLN), caudal (cLN), and axillary (axLN) (A), adipose tissue surrounding the iLNs (B), and in the liver, spleen, intestine, lungs, heart, and blood (C) [Healthy + Luci-eASCs intranodal (IN), $n=12$; TNBS + Luci-eASCs IN $n=25$ ] were expressed as percentage of light units per tissue relative to the total number of light units per mouse. Data are presented by dots and box-plots that represent the interquartile range (p75, upper edge; p25, lower edge; p50, midline; p95, line above the box, and p5, line below the box) of the percentage of total bioluminescence signal. Significance was analyzed by the Mann-Whitney $U$ test and represented by ${ }^{\star} p<0.05$, ${ }^{\star \star} p<0.01$, and ${ }^{\star \star \star} p<0.001$. Results correspond to four independent experiments.
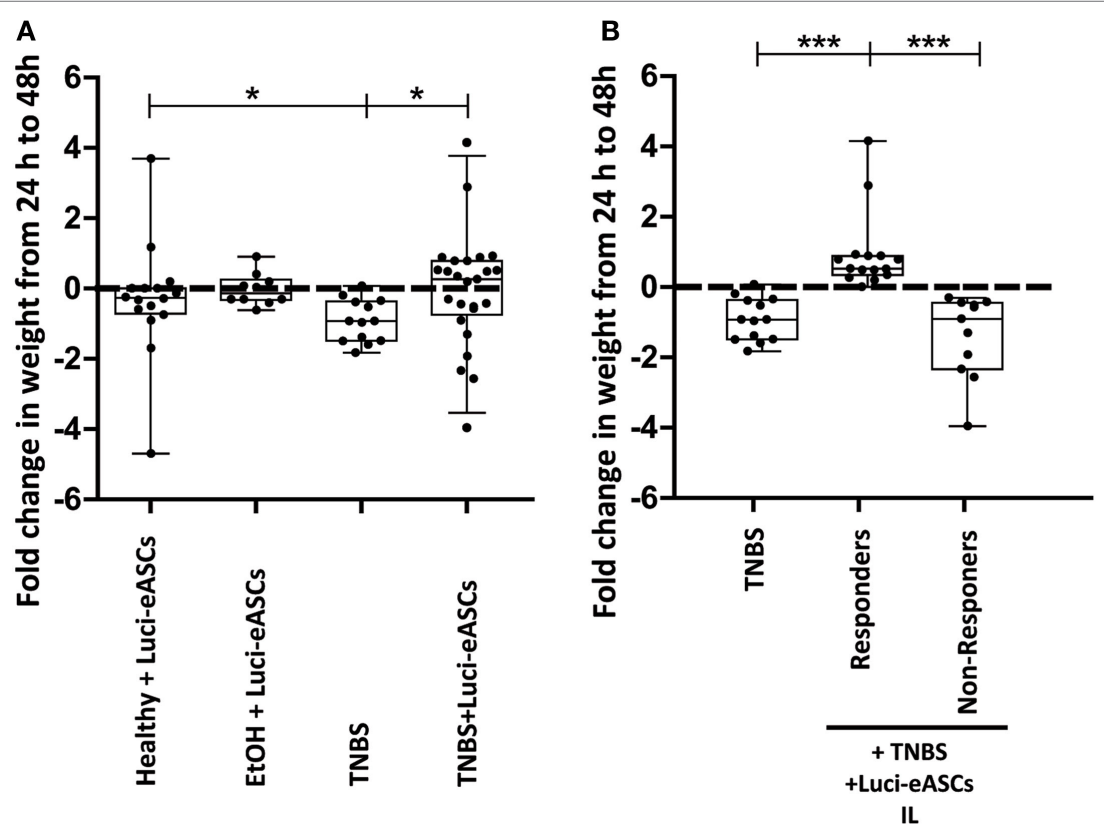

FIGURE 3 | Body weight changes in healthy and TNBS-colitic mice. Fold change in body weight from 24 to $48 \mathrm{~h}$ after intranodal (IN) administration are represented. (A) As controls, mice infused with the Luci-eASC IN with or without intrarectal 50\% EtOH (vehicle of the TNBS). Healthy + Luci-eASCs IN, $n=16$; EtOH + LucieASCs, $n=10$; TNBS, $n=14$; TNBS + Luci-eASCs IN = 26. (B) Stratification of in vivo responses to treatment with Luci-eASCs administration IN in TNBS-colitic mice according to the fold change in body weights from 24 to $48 \mathrm{~h}$. Colitic mice treated with Luci-eASCs were grouped into "responders" (Rs mice that did not lose weight at $48 \mathrm{~h}$ in comparison with $24 \mathrm{~h}$ ) and "non-responders" (NRs mice that lost weight at $48 \mathrm{~h}$ in comparison with $24 \mathrm{~h}$ ). TNBS, $n=14$; TNBS + Luci-eASCs IN Rs, $n=15$ and TNBS + Luci-eASCs IN NRs, $n=11$. Data are presented by dots and box-plots that represent the interquartile range (p75, upper edge; p25, lower edge; p50, midline; p95, line above the box; and p5, line below the box) of the weight fold change at $48 \mathrm{~h}$ in comparison with the day 0 . Significance was analyzed by the Mann-Whitney $U$ test and represented by ${ }^{*} p<0.05$ and ${ }^{* * *} p<0.001$. Results correspond to four independent experiments.

iLNs. Only $0.4(0.2-1.3) \%$ of the bioluminescence signal was found in the rest of LNs analyzed with no clear preference for any of them. Although the amount of Luci-eASCs that migrate to the other tissues and organs analyzed was very low, there was a significant increase in the bioluminescence signal in the intestine in TNBS-colitic mice.

\section{Expanded Adipose-Derived MSC Administration by the IN Route Protects against TNBS-Induced Colitis}

To investigate if the IN administration of Luci-eASCs can modulate acute inflammation, colitis was induced by administration of TNBS. One hour after the inflammatory challenge was induced, Luci-eASCs were infused IN. Body weights of mice were monitored within the first $48 \mathrm{~h}$ as the most meaningful physiological parameter for monitoring acute intestinal inflammation. As shown in Figure 3A, TNBS-treated mice had a significant reduction in their body weights $[-0.92(-1.49$ to -0.36$)]$ fold weight change in comparison with control mice [healthy mice, -0.26 ( -0.71 to 0.00$)$ fold body weight change and mice treated with intrarectal $50 \% \mathrm{EtOH}$ as the vehicle for the TNBS, -0.13 ( -0.32 to 0.25 ) fold body weight change]. The group of colitic mice treated with Luci-eASCs did not lose weight at $48 \mathrm{~h}$ after the inflammatory challenge $[0.26(-0.74$ to 0.79$)]$ in comparison to the untreated colitic mice.

These results demonstrate that, for the first time, the IN administration of eASCs is feasible and that can modulate efficiently acute intestinal inflammatory responses.

\section{Increased Amount of Luci-eASCs Within the Lymphatic System Can Be Correlated with an Improved Immunomodulation}

To investigate whether a correlation between the biodistribution of the Luci-eASCs and the modulation of the inflammation exists, 
we used body weight changes between 24 and 48 h postinfusion of the Luci-eASCs as a parameter to stratify mouse responses to treatment with Luci-eASCs. In this sense, "responder" mice were those TNBS-colitic mice that did not lose, or even gained, weight between 24 and 48 h postinfusion of the Luci-eASCs [0.53 (0.34-0.89) in comparison with the TNBS-colitic mice, -0.92 $(-1.49$ to -0.36$)]$. "Non-responder" mice were those TNBScolitic mice that continued to lose weight $48 \mathrm{~h}$ after the infusion of the Luci-eASCs $[-0.91(-2.34$ to -0.45$)]$ in comparison with the colitic mice. According to this stratification criteria, when LucieASCs were administered IN, 15 out of the 26 mice did not lose weight (responder mice). This means that $58 \%$ of mice treated with Luci-eASCs had a positive response to the treatment with Luci-eASCs (Figure 3B).

Additionally, we compared the bioluminescence signals in the tissues and organs and in the LNs in the "responder" and "non-responder" mice. As shown in Figure 4, no differences in the total bioluminescence signal were found neither in the LNs [56 (49.1-87.0)\% in responder mice and $58.4(46.1-77.1) \%$ in non-responder mice] nor in the other tissues and organs studied

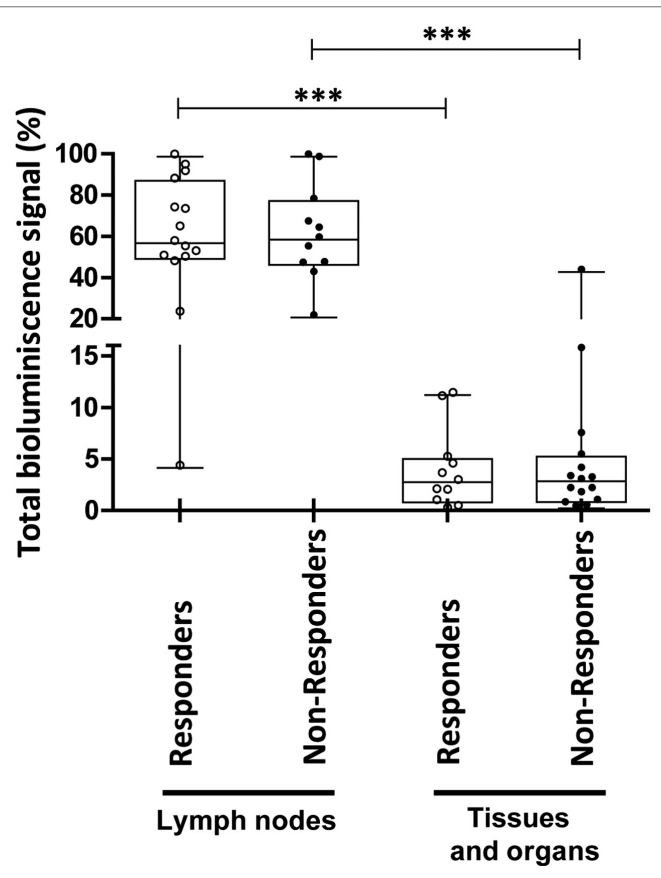

FIGURE 4 | Analysis of total bioluminescence signal of Luci-eASCs in lymph nodes (LNs) and in tissues and organs at $48 \mathrm{~h}$ in "responder" and "non-responder" TNBS-colitic mice treated with Luci-eASCs. Sum of bioluminescence signals measured at $48 \mathrm{~h}$ as percentage of light units per LN (inguinal, popliteal, parathymic, parathyroid, mesenteric, caudal, and axillary included) and per each tissue and organ (liver, spleen, intestine, lungs, heart and blood included) relative to the total number of light units per mouse were analyzed. Healthy + Luci-eASCs intranodal (IN), $n=13$; TNBS + Luci-eASCs IN Rs $n=15$; TNBS + Luci-eASCs IN NRs $n=11$. Data are presented by dots and box-plots that represent the interquartile range (p75, upper edge; p25, lower edge; p50, midline; p95, line above the box; and p5, line below the box) of the percentage of total bioluminescence signal. Significance was analyzed by the Mann-Whitney $U$ test and represented by ${ }^{\star \star \star} p<0.001$. Results correspond to four independent experiments.
[2.8 $(0.8-5.2) \%$ in responder mice and $2.8(0.8-5.0) \%$ in nonresponder mice], suggesting the lack of correlation between the modulation of acute inflammation in the intestine and the bioluminescence signal in LNs and other tissues and organs.

When the bioluminescence signal of Luci-eASCs was analyzed at the injection site, no significant differences were found between the responder and the non-responder mice, neither in the iLNs [50.8 (43.1-67.0)\% for responders; 56.0 (40.0-72.3)\% for nonresponder mice] nor in the iLN-adipose tissue [32.3 (9.2-47.0)\% for the responder mice, $40.0(22.1-48.5) \%$ for the non-responder mice, Figures 5A,B].

In contrast to this, the bioluminescence signals were tended to increase in the popliteal and parathyroid LNs of the responder mice in comparison with the non-responder mice $[0.7(0.3-1.4)$ vs. $0.2(0.1-0.5) \%$ in popliteal and $0.9(0.5-1.8)$ vs. $0.4(0.2-0.6) \%$ in the thyroid LNs, Figure 5A]. Also, the bioluminescence signal increased, but not significantly, in the parathymic and mesenteric LNs of the responder mice in comparison with the non-responder mice $[0.56(0.17-2.92)$ vs. $0.28(0.10-0.97) \%$ in parathymic and $1.32(0.66-2.920)$ vs. $0.44(0.34-0.76) \%$ in the mesenteric LNs, Figure 5A]. This suggests that, in some instances, the increase amount of Luci-eASCs within the lymphatic system can be correlated with a better immunomodulation of the inflammatory responses.

However, no clear differences were found in the bioluminescence signals in tissues and organs analyzed between responder and non-responder mice. Although, a tendency to increase the bioluminescence signal was found in the liver, spleen, lungs, heart and blood of responder mice in comparison with the non-responder mice. Also, there was a significant increase in the bioluminescence signal in the liver [0.37 $(0.10-1.26) \%]$ in comparison with the heart $[0.17(0.03-0.30) \%]$ of the responder mice but not in the non-responder mice $[0.05(0.03-0.57)$ vs. 0.090 (0.002-0.299)\%, Figure 5C].

Overall, these data indicate that, in responder mice, an increase amount of Luci-eASCs is located within the lymphatic system as suggested by the increase bioluminescence signals found in the popliteal, parathymic, parathyroid, and mesenteric LNs in comparison with the non-responder mice. However, in the tissues and organs analyzed these differences were not observed, although increased bioluminescence signals were found in the inflamed intestine.

\section{DISCUSSION}

The functional properties of the MSCs have become of great interest for the treatment of a variety of diseases $(20,47,48)$. At present, it is unknown whether the therapeutic effects of MSCs are carried out by a small fraction of the administered MSCs that migrates to sites of inflammation or/and through distant induction of regulatory immune cells that, rapidly and subsequently, mediate immunomodulation and tissue repair (22). In any case, their therapeutic effects are linked to a final increase of myeloid and lymphoid cells with a regulatory phenotype in the secondary lymphoid tissues associated to the inflammation site $(18,19,25,26)$. Thus, we proposed, for the first time, the administration of eASCs directly into the LNs, in order to determine 


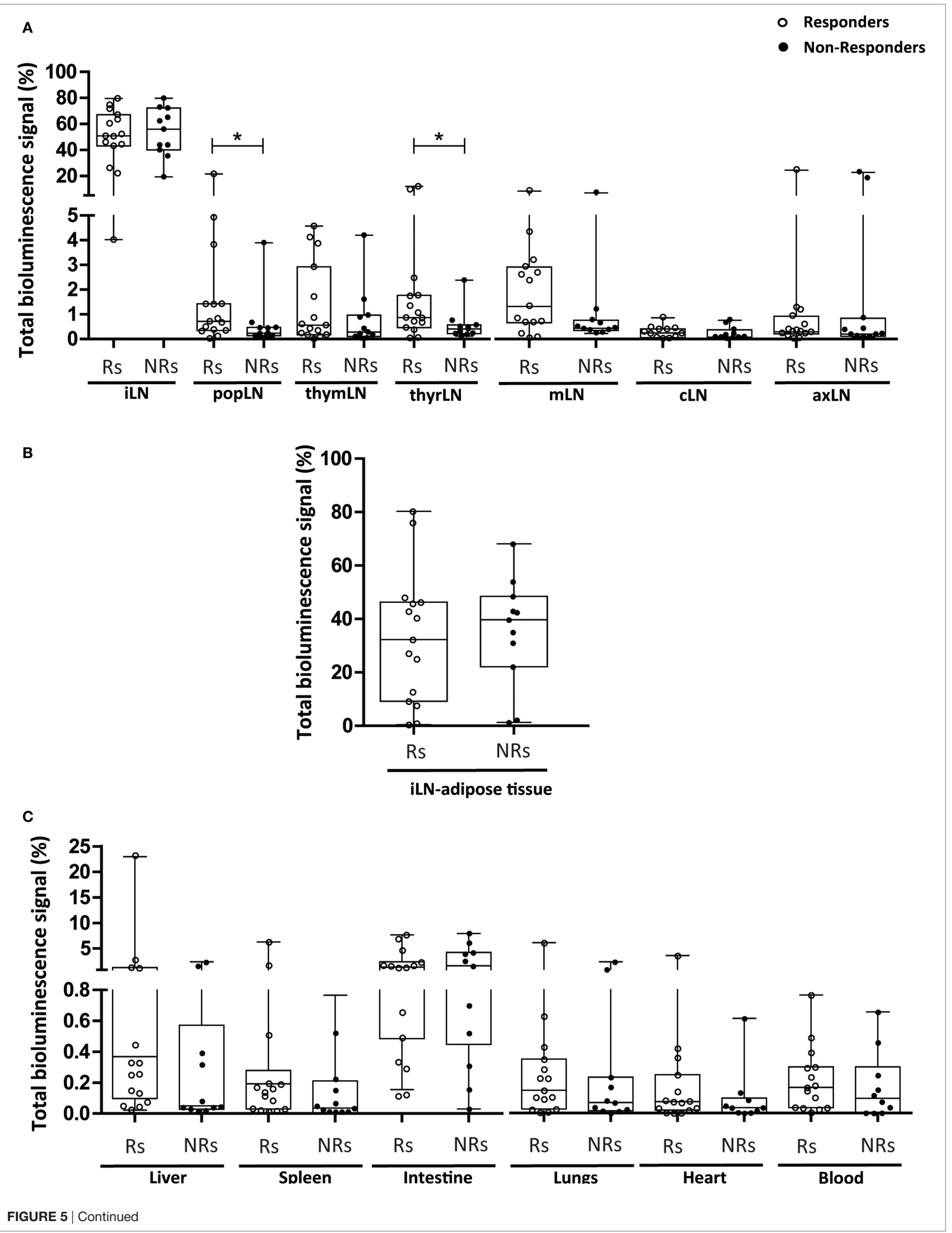




\section{FIGURE 5 | Continued}

Analysis of total bioluminescence signal of Luci-eASCs in lymph nodes (LNs) (inguinal, popliteal, parathymic, parathyroid, mesenteric, caudal, and axillary), adipose tissue adjacent the inguinal LNs and in tissues and organs (the liver, spleen, intestine, lungs, heart, and blood) in "responder" and "non-responder" TNBS-colitic mice treated with Luci-eASCs at $48 \mathrm{~h}$. Bioluminescence signals, measured at $48 \mathrm{~h}$ in the inguinal LNs (iLN), popliteal (popLN), parathymic (thymLN), parathyroid (thyrLN), mesenteric $(\mathrm{mLN})$, caudal $(\mathrm{cLN})$, and axillary $(\operatorname{axLN})(\mathbf{A})$, the adipose tissue surrounding the iLNs (B), and in the liver, spleen, intestine, lungs, heart, and blood (C) in "responders" mice (that did not lose weight from 24 to $48 \mathrm{~h}$ ) and "non-responders" mice (that lost weight from 24 to $48 \mathrm{~h}$ ) were analyzed as percentage of light units per tissue relative to the total number of light units per mouse. Healthy + Luci-eASCs intranodal (IN), $n=12$; TNBS + Luci-eASCs IN Rs $n=15$; TNBS + Luci-eASCs IN NRs $n=11$. Data are presented by dots and box-plots that represent the interquartile range (p75, upper edge; p25, lower edge; p50, midline; p95, line above the box; and p5, line below the box) of the percentage of total bioluminescence signal. Significance was analyzed by the Mann-Whitney $U$ test and represented by ${ }^{*} p<0.05$. Results correspond to four independent experiments.

whether the IN administration of MSCs could be an alternative strategy to modulate inflammation. The IN (or intralymphatic) route is being extensively used in immunotherapy protocols for treatment of cancer (29-32) and allergy $(33,34)$. The data reported indicate that this route of administration is feasible and can be translated to the clinic (35-37). Additionally, Gil-Ortega et al. provided robust evidences that endogenous adipose stromal cells can traffic in vivo through lymphatic system and home to the LNs (28).

In this study, we observed that IN administration of LucieASCs can modulate acute intestinal inflammation in a mouse model of colitis induced by TNBS as shown by the maintenance of the body weights of colitic mice at $48 \mathrm{~h}$ after infusion of the cells (Figure 3). To our knowledge, this is the first time that a single dose of $3.2 \times 10^{5}$ eASCs show immunomodulatory effects in a mouse model of colitis induced by TNBS (10, 40, 49-52). We cannot provide any reasonable explanation for the observation that $58 \%$ of eASC-treated mice have a positive response to the cell therapy. It should be noticed that the biodistribution analysis was conducted using a single IL infusion of the Luci-eASCs whereas the majority of studies aiming at modulating immune responses used multiple eASCs doses to achieve robust modulation of inflammation. Since the main goal of our study was to define the in vivo biodistribution of eASCs infused intranodally in an inflammatory environment, we cannot exclude that multiple IL infusion of eASCs would achieve a complete response.

Furthermore, Mancheño-Corvo et al. demonstrated that eASCs administered IN reduced systemic inflammation, in an experimental model of arthritis, by inducting CD $25^{+}$Foxp $3^{+} \mathrm{CD} 4^{+}$ cells and $\mathrm{IL} 10^{+} \mathrm{CD} 4^{+}$cells in the LNs and in the spleen (27).

In vivo biodistribution of MSCs is an important parameter to take into account when evaluating the efficacy of the cell therapy with MSCs. After intravenous administration, the most widely used route for MSCs in the clinic, the infused MSCs accumulate preferential in the lungs $(24,38)$ and this is generally accompanied by a transient immunomodulatory effect $(22,26,53-56)$ due to their short-term persistence in vivo regardless of their origin (syngeneic, allogeneic or, even, xenogeneic). This, obviously, might impact the bioavailability of the cells, their targeting to tissues and, probably, their long-term therapeutic efficacy (24). Thus, in this study, we proposed to study the biodistribution of the IN administration of MSCs which potentially may represent an alternative route of administration for MSCs. The number of MSCs that can be delivered inside the LN after IN administration is significantly greater than the number of cells that reach the LNs following their delivery through the blood stream. This may allow achieving therapeutic effects using a low dose of MSCs. This will have major implications to improve the safety of the cell therapy together with a significant reduction in the production costs.

In our study, we observed that, following the IN administration of the Luci-eASCs, the majority of the bioluminescence signal was found in the LNs (Figure 1) mainly at the injection site, either in the inguinal LNs or in the adipose tissue surrounding the iLNs where the cells were injected (Figures 2A,B; Figure S2C in Supplementary Material). Only $0.4(0.2-1.3) \%$ of the bioluminescence signal was found in other locations within the lymphatic system with no clear preference for any of the LNs analyzed (Figure 2A). These results show that, by the IN administration of MSCs, a higher number of cells are within the lymphatic system when compared to intravenous administration $(25,41)$ which could improve their therapeutic effects.

Only $1.9(0.4-4.1) \%$ of the bioluminescence signal was found in the tissues and organs analyzed and, surprisingly, in TNBScolitic mice, a significant increase of the total bioluminescence signal was found with in comparison with the healthy mice. This paralleled with a decrease in the bioluminescence signal in the iLNs and in the adipose tissue surrounding the iLNs of TNBScolitic mice when compared to healthy mice (Figures 1 and 2), suggesting a rapid and preferential trafficking of the Luci-eASCs to the inflamed tissues and organs upon their IN administration. This increase of the bioluminescence signal corresponded mainly to the intestine and to a lesser extent to the liver, spleen, lungs, and blood of the Luci-eASC-treated colitic mice suggesting that, by the IN route, MSCs have a preferential migration to the inflamed tissues, similar to what was happened when MSCs are infused by other routes of administration (23-25).

Trying to know if the presence of the MSCs at the inflammation site is necessary for the modulation of the immune responses, we investigated the correlation between the biodistribution of the Luci-eASCs and the modulation of the inflammation attending to the stratification criteria into "responders" and "non-responders" mice. These stratification criteria were done based on the body weights of the Luci-eASC-treated colitic mice. The increase amount of Luci-eASCs within the lymphatic system in the responder mice can be correlated with an improved immunomodulation (Figure 5). This increase amount of LucieASCs within the lymphatic system was preferentially found in the popLNs, thyrLNs, thymLNs, and mLNs. We did not see 
any correlation between the presence of the MSCs in the tissues and organs analyzed and the modulation of the inflammation, this result suggests that the presence of the Luci-eASCs in the intestine does not seem to be necessary to achieve an adequate modulation of the inflamed intestine.

In summary, all these results suggest that the presence of the Luci-eASCs within the lymphatic system is responsible for their immunomodulatory effects. The IN administration of the Luci-eASCs allows the trafficking of a small proportion of cells to the inflamed intestine; although, a direct correlation with their biological effects could not be established. Further, these results confirm our hypothesis that the immunomodulatory effects of the eASCs may take place in the LNs where the immunoresponses are orchestrated.

Based on these results and those reported in the accompanying manuscript (27), further investigations are warranted to assess the beneficial effect of the IN route in cell therapy protocols with eASCs for treatment of immunomediated disorders.

\section{CONCLUSION}

These data demonstrate the feasibility of using the IN route of administration to infuse eASCs for the treatment of intestinal inflammatory diseases since Luci-eASCs can modulate an acute intestinal inflammation.

After IN administration, the majority of the Luci-eASCs remained in the inguinal LNs where the cells were infused and only lower levels of bioluminescence signals were found in tissues and organs related to the inflammation. After intestinal inflammation, an increased trafficking of the Luci-eASCs to the inflamed organs was found. In some instances, the increase amount of Luci-eASCs within the lymphatic system can be correlated with an improved immunomodulation. Taken together, these results indicate that the IN administration of eASCs represents a novel route for administering eASCs in cell therapy protocols.

\section{ETHICS STATEMENT}

Institutional Animal Care and Use Committee at University of Albacete (Spain) and Spanish Ethics Committee of reference (Clinica de la Luz Hospital) for the site of tissue procurement.

\section{AUTHOR CONTRIBUTIONS}

Conception and/or design of the work: ML-S, OD, JA, DB, JR, JB, WD, EL, and MG. Acquisition of data for the work: ML-S, PM-C, AE, RM, JA, EL, and MG. Analysis and interpretation of data for the work: ML-S, PM-C, AE, RM, OD, JA, DB, JR, JB, WD, EL, and MG. Manuscript writing: ML-S, PM-C, and MG. Revised manuscript for important intellectual content: AE, RM, OD, DB, $\mathrm{JR}, \mathrm{JB}$, and WD. All authors reviewed the manuscript and gave final approval for the work.

\section{FUNDING}

European Community's 7th Framework Program for the collaborative project: "REGENER-AR: Bringing Regenerative Medicine into the market: Allogeneic eASCs Phase IB/IIA clinical trial for treating Rheumatoid Arthritis" (contract no. 279174 EC).

\section{SUPPLEMENTARY MATERIAL}

The Supplementary Material for this article can be found online at http://journal.frontiersin.org/article/10.3389/fimmu.2017.00638/ full\#supplementary-material.

FIGURE S1 | Characterization of Luci-eASCs. (A) Luci-eASCs were characterized by flow cytometry using antibodies against CD73, CD90, CD105, CD14, CD34, CD45, and HLA-DR (gray color). The corresponding isotype controls for each surface marker are shown (white color). Histograms shown are representative of three experiments. (B) Representative histograms of T-lymphocyte proliferation are shown. CFSE-labeled peripheral blood mononuclear cells (PBMCs) were stimulated with anti-CD3/CD2/CD28-coated beads in the absence (gray color) or presence (white color) of transduced ASCs (Luci-eASCs, left) or untransduced eASCs (right), ratio 1:25 eASCs or Luci-eASC:PBMCs. Proliferation of the viable CD3 ${ }^{+} \mathrm{T}$ cells was monitored after $120 \mathrm{~h}$ by flow cytometry.

FIGURE S2 | In vivo imaging of Luci-eASCs after IN infusion of Luci-eASCs. (A) Representative images of total bioluminescence signal at 24 and $48 \mathrm{~h}$ when Luci-eASCs were administered intranodally in healthy mice. (B) Graph represents the decline of total bioluminescence signal (luciferase activity) at different time points after $3.2 \times 10^{5}$ Luci-eASCs were administered intranodally into healthy mice. (C) Representative images of bioluminescence signal at $48 \mathrm{~h}$ of left inguinal (iLN), right inguinal lymph nodes (LNs), popliteal (popLN), parathyroid (thyrLN), parathymic (thymLN), mesenteric $(m L N)$, and axillary $(a x L N)$ lymph nodes. (D) Representative images of bioluminescence signal at $48 \mathrm{~h}$ in intestine, blood, lungs, heart, liver, spleen, and adipose tissue adjacent inguinal lymph nodes (iLNs AT).

\section{REFERENCES}

1. Chamberlain G, Fox J, Ashton B, Middleton J. Concise review: mesenchymal stem cells: their phenotype, differentiation capacity, immunological features, and potential for homing. Stem Cells (2007) 25(11):2739-49. doi:10.1634/ stemcells.2007-0197

2. English K, French A, Wood KJ. Mesenchymal stromal cells: facilitators of successful transplantation? Cell Stem Cell (2010) 7(4):431-42. doi:10.1016/j. stem.2010.09.009

3. Ding DC, Chang YH, Shyu WC, Lin SZ. Human umbilical cord mesenchymal stem cells: a new era for stem cell therapy. Cell Transplant (2015) 24(3):339-47. doi:10.3727/096368915X686841

4. Sessarego N, Parodi A, Podesta M, Benvenuto F, Mogni M, Raviolo V, et al. Multipotent mesenchymal stromal cells from amniotic fluid: solid perspectives

for clinical application. Haematologica (2008) 93(3):339-46. doi:10.3324/ haematol.11869

5. Gimble JM, Bunnell BA, Frazier T, Rowan B, Shah F, Thomas-Porch C, et al. Adipose-derived stromal/stem cells: a primer. Organogenesis (2013) 9(1):3-10. doi:10.4161/org.24279

6. Liu J, Yu F, Sun Y, Jiang B, Zhang W, Yang J, et al. Characteristics and potential applications of human dental tissue-derived mesenchymal stem cells. Stem Cells (2014) 33(3):627-38. doi:10.1002/stem.1909

7. Salem HK, Thiemermann C. Mesenchymal stromal cells: current understanding and clinical status. Stem Cells (2010) 28(3):585-96. doi:10.1002/ stem. 269

8. Griffin MD, Elliman SJ, Cahill E, English K, Ceredig R, Ritter T. Concise review: adult mesenchymal stromal cell therapy for inflammatory diseases: how well are we joining the dots? Stem Cells (2013) 31(10):2033-41. doi:10.1002/stem.1452 
9. Introna M, Rambaldi A. Mesenchymal stromal cells for prevention and treatment of graft-versus-host disease: successes and hurdles. Curr Opin Organ Transplant (2015) 20(1):72-8. doi:10.1097/MOT.0000000000000158

10. Chen QQ, Yan L, Wang CZ, Wang WH, Shi H, Su BB, et al. Mesenchymal stem cells alleviate TNBS-induced colitis by modulating inflammatory and autoimmune responses. World J Gastroenterol (2013) 19(29):4702-17. doi:10.3748/ wjg.v19.i29.4702

11. Martinez-Montiel Mdel P, Gomez-Gomez GJ, Flores AI. Therapy with stem cells in inflammatory bowel disease. World J Gastroenterol (2014) 20(5): 1211-27. doi:10.3748/wjg.v20.i5.1211

12. Nagaishi K, Arimura Y, Fujimiya M. Stem cell therapy for inflammatory bowel disease. J Gastroenterol (2015) 50(3):280-6. doi:10.1007/ s00535-015-1040-9

13. Constantin G, Marconi S, Rossi B, Angiari S, Calderan L, Anghileri E, et al. Adipose-derived mesenchymal stem cells ameliorate chronic experimental autoimmune encephalomyelitis. Stem Cells (2009) 27(10):2624-35. doi:10.1002/stem.194

14. Liang J, Zhang H, Hua B, Wang H, Lu L, Shi S, et al. Allogenic mesenchymal stem cells transplantation in refractory systemic lupus erythematosus: a pilot clinical study. Ann Rheum Dis (2010) 69(8):1423-9. doi:10.1136/ ard.2009.123463

15. Wang D, Zhang H, Liang J, Li X, Feng X, Wang H, et al. Allogeneic mesenchymal stem cell transplantation in severe and refractory systemic lupus erythematosus: 4 years of experience. Cell Transplant (2013) 22(12):2267-77. doi:10.3727/096368911X582769

16. MacDonald GI, Augello A, De Bari C. Role of mesenchymal stem cells in reestablishing immunologic tolerance in autoimmune rheumatic diseases. Arthritis Rheum (2011) 63(9):2547-57. doi:10.1002/art.30474

17. Wang L, Wang L, Cong X, Liu G, Zhou J, Bai B, et al. Human umbilical cord mesenchymal stem cell therapy for patients with active rheumatoid arthritis: safety and efficacy. Stem Cells Dev (2013) 22(24):3192-202. doi:10.1089/ scd.2013.0023

18. Lopez-Santalla M, Mancheno-Corvo P, Menta R, Lopez-Belmonte J, DelaRosa O, Bueren JA, et al. Human adipose-derived mesenchymal stem cells modulate experimental autoimmune arthritis by modifying early adaptive T cell responses. Stem Cells (2015) 33(12):3493-503. doi:10.1002/stem.2113

19. Lopez-Santalla M, Menta R, Mancheno-Corvo P, Lopez-Belmonte J, DelaRosa O, Bueren JA, et al. Adipose-derived mesenchymal stromal cells modulate experimental autoimmune arthritis by inducing an early regulatory innate cell signature. Immun Inflamm Dis (2016) 4(2):213-24. doi:10.1002/iid3.106

20. Alvaro-Gracia JM, Jover JA, Garcia-Vicuna R, Carreno L, Alonso A, Marsal S, et al. Intravenous administration of expanded allogeneic adipose-derived mesenchymal stem cells in refractory rheumatoid arthritis (Cx611): results of a multicentre, dose escalation, randomised, single-blind, placebo-controlled phase Ib/IIa clinical trial. Ann Rheum Dis (2017) 76(1):196-202. doi:10.1136/ annrheumdis-2015-208918

21. Yagi H, Soto-Gutierrez A, Parekkadan B, Kitagawa Y, Tompkins RG, Kobayashi N, et al. Mesenchymal stem cells: mechanisms of immunomodulation and homing. Cell Transplant (2010) 19(6):667-79. doi:10.3727/0963 68910X508762

22. Eggenhofer E, Luk F, Dahlke MH, Hoogduijn MJ. The life and fate of mesenchymal stem cells. Front Immunol (2014) 19(5):148. doi:10.3389/ fimmu. 2014.00148

23. Assis AC, Carvalho JL, Jacoby BA, Ferreira RL, Castanheira P, Diniz SO, et al. Time-dependent migration of systemically delivered bone marrow mesenchymal stem cells to the infarcted heart. Cell Transplant (2010) 19(2):219-30. doi:10.3727/096368909X479677

24. Eggenhofer E, Benseler V, Kroemer A, Popp FC, Geissler EK, Schlitt HJ, et al. Mesenchymal stem cells are short-lived and do not migrate beyond the lungs after intravenous infusion. Front Immunol (2012) 26(3):297. doi:10.3389/ fimmu.2012.00297

25. Lee HJ, Ko JH, Jeong HJ, Ko AY, Kim MK, Wee WR, et al. Mesenchymal stem/ stromal cells protect against autoimmunity via CCL2-dependent recruitment of myeloid-derived suppressor cells. J Immunol (2015) 194(8):3634-45. doi:10.4049/jimmunol.1402139

26. Gonzalez MA, Gonzalez-Rey E, Rico L, Buscher D, Delgado M. Treatment of experimental arthritis by inducing immune tolerance with human adipose-derived mesenchymal stem cells. Arthritis Rheum (2009) 60(4):100619. doi:10.1002/art.24405

27. Mancheno-Corvo P, Lopez-Santalla M, Menta R, DelaRosa O, Mulero F, Del Rio B, et al. Intralymphatic administration of adipose mesenchymal stem cells reduces the severity of collagen-induced experimental arthritis. Front Immunol (2017) 8:462. doi:10.3389/fimmu.2017.00462

28. Gil-Ortega M, Garidou L, Barreau C, Maumus M, Breasson L, Tavernier G, et al. Native adipose stromal cells egress from adipose tissue in vivo: evidence during lymph node activation. Stem Cells (2013) 31(7):1309-20. doi:10.1002/ stem. 1375

29. Gilliet M, Kleinhans M, Lantelme E, Schadendorf D, Burg G, Nestle FO. Intranodal injection of semimature monocyte-derived dendritic cells induces T helper type 1 responses to protein neoantigen. Blood (2003) 102(1):36-42. doi:10.1182/blood-2002-07-2274

30. Maier T, Tun-Kyi A, Tassis A, Jungius KP, Burg G, Dummer R, et al. Vaccination of patients with cutaneous T-cell lymphoma using intranodal injection of autologous tumor-lysate-pulsed dendritic cells. Blood (2003) 102(7):2338-44. doi:10.1182/blood-2002-08-2455

31. Cai S, Xie Y, Davies NM, Cohen MS, Forrest ML. Pharmacokinetics and disposition of a localized lymphatic polymeric hyaluronan conjugate of cisplatin in rodents. J Pharm Sci (2010) 99(6):2664-71. doi:10.1002/jps.22016

32. Kreiter S, Selmi A, Diken M, Koslowski M, Britten CM, Huber C, et al. Intranodal vaccination with naked antigen-encoding RNA elicits potent prophylactic and therapeutic antitumoral immunity. Cancer Res (2010) 70(22):9031-40. doi:10.1158/0008-5472.CAN-10-0699

33. Johansen P, Mohanan D, Martinez-Gomez JM, Kundig TM, Gander B. Lympho-geographical concepts in vaccine delivery. J Control Release (2010) 148(1):56-62. doi:10.1016/j.jconrel.2010.05.019

34. Mohanan D, Slutter B, Henriksen-Lacey M, Jiskoot W, Bouwstra JA, Perrie Y, et al. Administration routes affect the quality of immune responses: a cross-sectional evaluation of particulate antigen-delivery systems. J Control Release (2010) 147(3):342-9. doi:10.1016/j.jconrel.2010.08.012

35. Senti G, Prinz Vavricka BM, Erdmann I, Diaz MI, Markus R, McCormack SJ, et al. Intralymphatic allergen administration renders specific immunotherapy faster and safer: a randomized controlled trial. Proc Natl Acad Sci U S A (2008) 105(46):17908-12. doi:10.1073/pnas.0803725105

36. Casale TB, Stokes JR. Immunotherapy: what lies beyond. JAllergy Clin Immunol (2014) 133(3):612-9. doi:10.1016/j.jaci.2014.01.007

37. Senti G, Kundig TM. Intralymphatic immunotherapy. World Allergy Organ J (2015) 8(1):9. doi:10.1186/s40413-014-0047-7

38. Fischer UM, Harting MT, Jimenez F, Monzon-Posadas WO, Xue H, Savitz SI, et al. Pulmonary passage is a major obstacle for intravenous stem cell delivery: the pulmonary first-pass effect. Stem Cells Dev (2009) 18(5):683-92. doi:10.1089/scd.2008.0253

39. Ramot Y, Meiron M, Toren A, Steiner M, Nyska A. Safety and biodistribution profile of placental-derived mesenchymal stromal cells (PLX-PAD) following intramuscular delivery. Toxicol Pathol (2009) 37(5):606-16. doi:10.1177/0192623309338383

40. Castelo-Branco MT, Soares ID, Lopes DV, Buongusto F, Martinusso CA, do Rosario A Jr, et al. Intraperitoneal but not intravenous cryopreserved mesenchymal stromal cells home to the inflamed colon and ameliorate experimental colitis. PLoS One (2012) 7(3):e33360. doi:10.1371/journal. pone. 0033360

41. Toupet K, Maumus M, Peyrafitte JA, Bourin P, van Lent PL, Ferreira R, et al. Long-term detection of human adipose-derived mesenchymal stem cells after intraarticular injection in SCID mice. Arthritis Rheum (2013) 65(7):1786-94. doi:10.1002/art.37960

42. Cerri S, Greco R, Levandis G, Ghezzi C, Mangione AS, Fuzzati-Armentero MT, et al. Intracarotid infusion of mesenchymal stem cells in an animal model of Parkinson's disease, focusing on cell distribution and neuroprotective and behavioral effects. Stem Cells Transl Med (2015) 4(9):1073-85. doi:10.5966/ sctm.2015-0023

43. Dominici M, Le Blanc K, Mueller I, Slaper-Cortenbach I, Marini F, Krause D, et al. Minimal criteria for defining multipotent mesenchymal stromal cells. The International Society for Cellular Therapy position statement. Cytotherapy (2006) 8(4):315-7. doi:10.1080/14653240600855905

44. DelaRosa O, Lombardo E, Beraza A, Mancheno-Corvo P, Ramirez C, Menta R, et al. Requirement of IFN-gamma-mediated indoleamine 2,3-dioxygenase 
expression in the modulation of lymphocyte proliferation by human adiposederived stem cells. Tissue Eng Part A (2009) 15(10):2795-806. doi:10.1089/ten. TEA.2008.0630

45. Menta R, Mancheno-Corvo P, Del Rio B, Ramirez C, DelaRosa O, Dalemans W, et al. Tryptophan concentration is the main mediator of the capacity of adipose mesenchymal stromal cells to inhibit T-lymphocyte proliferation in vitro. Cytotherapy (2014) 16(12):1679-91. doi:10.1016/j.jcyt.2014.07.004

46. Bramhall M, Florez-Vargas O, Stevens R, Brass A, Cruickshank S. Quality of methods reporting in animal models of colitis. Inflamm Bowel Dis (2015) 21(6):1248-59. doi:10.1097/MIB.0000000000000369

47. Garcia-Olmo D, Garcia-Arranz M, Herreros D, Pascual I, Peiro C, Rodriguez-Montes JA. A phase I clinical trial of the treatment of Crohn's fistula by adipose mesenchymal stem cell transplantation. Dis Colon Rectum (2005) 48(7):1416-23. doi:10.1007/s10350-005-0052-6

48. Perez-Simon JA, Lopez-Villar O, Andreu EJ, Rifon J, Muntion S, Diez Campelo M, et al. Mesenchymal stem cells expanded in vitro with human serum for the treatment of acute and chronic graft-versus-host disease: results of a phase I/II clinical trial. Haematologica (2011) 96(7):1072-6. doi:10.3324/ haematol.2010.038356

49. Ando $\mathrm{Y}$, Inaba M, Sakaguchi $\mathrm{Y}$, Tsuda M, Quan GK, Omae M, et al. Subcutaneous adipose tissue-derived stem cells facilitate colonic mucosal recovery from 2,4,6-trinitrobenzene sulfonic acid (TNBS)-induced colitis in rats. Inflamm Bowel Dis (2008) 14(6):826-38. doi:10.1002/ibd.20382

50. Duijvestein M, Wildenberg ME, Welling MM, Hennink S, Molendijk I, van Zuylen VL, et al. Pretreatment with interferon-gamma enhances the therapeutic activity of mesenchymal stromal cells in animal models of colitis. Stem Cells (2011) 29(10):1549-58. doi:10.1002/stem.698

51. Parekkadan B, Upadhyay R, Dunham J, Iwamoto Y, Mizoguchi E, Mizoguchi A, et al. Bone marrow stromal cell transplants prevent experimental enterocolitis and require host CD11b+ splenocytes. Gastroenterology (2011) 140(3):966-75. doi:10.1053/j.gastro.2010.10.013
52. Xie M, Qin H, Luo Q, He X, He X, Lan P, et al. Comparison of adipose-derived and bone marrow mesenchymal stromal cells in a murine model of Crohn's disease. Dig Dis Sci (2017) 62(1):115-23. doi:10.1007/s10620-016-4166-6

53. Gonzalez-Rey E, Anderson P, Gonzalez MA, Rico L, Buscher D, Delgado M. Human adult stem cells derived from adipose tissue protect against experimental colitis and sepsis. Gut (2009) 58(7):929-39. doi:10.1136/gut. 2008.168534

54. Gonzalez MA, Gonzalez-Rey E, Rico L, Buscher D, Delgado M. Adiposederived mesenchymal stem cells alleviate experimental colitis by inhibiting inflammatory and autoimmune responses. Gastroenterology (2009) 136(3):978-89. doi:10.1053/j.gastro.2008.11.041

55. Ankrum JA, Ong JF, Karp JM. Mesenchymal stem cells: immune evasive, not immune privileged. Nat Biotechnol (2014) 32(3):252-60. doi:10.1038/nbt.2816

56. Choi EW, Lee HW, Shin S, Park JH, Yun TW, Youn HY, et al. Comparative efficacies of long-term serial transplantation of syngeneic, allogeneic, xenogeneic, or CTLA4Ig overproducing xenogeneic adipose tissue-derived mesenchymal stem cells on murine systemic lupus erythematosus. Cell Transplant (2016) 25(6):1193-206. doi:10.3727/096368915X689442

Conflict of Interest Statement: PM-C, RM, OD, WD, and EL are full-time employees of TiGenix, DB is full-time employee of Grifols, and JA is full-time employee of Coretherapix.

Copyright $\odot 2017$ Lopez-Santalla, Mancheño-Corvo, Escolano, Menta, DelaRosa, Abad, Büscher, Redondo, Bueren, Dalemans, Lombardo and Garin. This is an open-access article distributed under the terms of the Creative Commons Attribution License (CC BY). The use, distribution or reproduction in other forums is permitted, provided the original author(s) or licensor are credited and that the original publication in this journal is cited, in accordance with accepted academic practice. No use, distribution or reproduction is permitted which does not comply with these terms. 